\title{
Éric Szulman, La navigation intérieure sous l'Ancien Régime. Naissance d'une politique publique
}

Rennes, PUR, 2014

\section{Stéphane Blond}

\section{(2) OpenEdition}

Journals

Édition électronique

URL : http://journals.openedition.org/artefact/560

DOI : $10.4000 /$ artefact.560

ISSN : 2606-9245

Éditeur :

Association Artefact. Techniques histoire et sciences humaines, Presses universitaires du Midi

Édition imprimée

Date de publication : 1 octobre 2016

Pagination : 427-429

ISBN : 978-2-7535-5174-9

ISSN : 2273-0753

Référence électronique

Stéphane Blond, «Éric Szulman, La navigation intérieure sous l'Ancien Régime. Naissance d'une politique publique », Artefact [En ligne], 4 | 2016, mis en ligne le 07 juillet 2017, consulté le 24 septembre 2020 URL : http://journals.openedition.org/artefact/560 ; DOI : https://doi.org/10.4000/artefact.560

Ce document a été généré automatiquement le 24 septembre 2020.

Artefact, Techniques, histoire et sciences humaines est mise à disposition selon les termes de la Licence Creative Commons Attribution - Pas d'Utilisation Commerciale - Pas de Modification 4.0 International. 


\section{Éric Szulman, La navigation intérieure sous l'Ancien Régime. Naissance d'une politique publique}

Rennes, PUR, 2014

Stéphane Blond

\section{RÉFÉRENCE}

Éric Szulman, La navigation intérieure sous l'Ancien Régime. Naissance d'une politique publique, Rennes, PUR, 2014, 378 p.

1 À travers l'ouvrage issu de sa thèse de doctorat soutenue en 2011, Éric Szulman donne à lire l'histoire de la navigation intérieure au cours des deux derniers siècles de l'Ancien Régime. Souvent abordée de manière indirecte, via les multiples études qui s'intéressent aux projets et aux travaux de canaux, cette question historique est peu abordée par le haut, autour de la réflexion des grands administrateurs et des multiples relais décisionnels.

2 Comme l'indique le sous-titre, la démarche de l'auteur insiste sur la «naissance d'une politique publique ", les administrateurs de la monarchie française faisant de la navigation un champ incontournable de l'intervention régalienne. L'originalité de l'étude procède de la description de l'émergence d'une notion de réseau, souvent plus tardive qu'avec les voies terrestres, même si les liens entre ces deux modes de transport sont forts. Si le mot « réseau » n'existe pas pendant l'Ancien Régime, souvent remplacé par l'expression systémique de «navigation intérieure », la notion et l'idée d'un réseau sont bien là.

3 Grâce à une méthode consistant à historiciser l'action publique, Éric Szulman se focalise sur les démarches opérées. Il s'intéresse à la multiplicité des actions qui fusionnent lentement en une action globale. En outre, contrairement à son postulat de départ, il ne néglige pas les acteurs. En effet, la définition du rôle du prince dans la 
politique de navigation passe constamment par l'examen des philosophies administratives, des rouages politiques, des cercles d'experts et de techniciens, mais aussi des sphères d'influence et de clientèle. S'appuyant sur la gigantesque correspondance encore conservée dans les archives et entretenue par de multiples intervenants, cette histoire s'appuie sur autant d'études de cas parfaitement maîtrisées qui permettent de saisir la politique globale.

4 L'ouvrage se structure autour de huit chapitres, avec un découpage chronologique auquel répondent des évolutions dans l'approche administrative. Le chapitre premier traite ainsi de l'émergence à la fin du XVII siècle d'une histoire intellectuelle de la navigation intérieure. Colbert et Vauban font partie de ceux qui approchent l'idée d'un système et le principe d'interventions coordonnées, se voulant plus efficaces et utiles aux pouvoirs publics. En effet, le rôle central revient à l'État, avec des enjeux commerciaux et militaires évoqués à travers des projets emblématiques et leurs nombreux avatars, comme le canal du Midi et le canal de Bourgogne.

5 Le chapitre 2 aborde la multiplication des projets et des initiatives privées à partir des années 1740. Cette inflexion sensible induit la mise en place d'un circuit administratif d'expertise au sein duquel l'enquête administrative constitue souvent un élément déclencheur, pour ne pas dire incitateur. Les théoriciens en appellent quant à eux à l'État, mais les moyens de celui-ci restent faibles. Les réseaux scientifiques et marchands tiennent aussi une place centrale, avec l'apparition concomitante d'une «science du commerce ».

6 Avec l'œuvre de Bertin décortiquée dans le chapitre 3, Éric Szulman décrit un moment singulier en matière d'intervention publique en faveur des voies navigables. De 1763 à 1773, en lien avec Parent, le ministre échafaude un système élaboré, construit autour d'un cercle vertueux pour le développement de l'économie du royaume. Pour autant, système ne veut pas dire réseau et pour concrétiser celui-ci la question du financement reste au cœur des débats. Le libéral Bertin pourfend à la fois le système des concessions et des péages, mais l'enquête de 1764 dégage peu de marges de manœuvre à l'État pour le lancement de travaux de construction, mais aussi l'entretien des voies navigables existantes.

7 Finalement, c'est au ministre Turgot que revient la mise en application et le développement du "plan Bertin» (chapitre 4). Avec Turgot, la question du financement aboutit, grâce à la conception d'une imposition unique qui rejoint sa réforme de la corvée pour les routes. Le ministre s'intéresse également à la police de la navigation, un aspect essentiel à l'efficacité du système, mais peu réformé depuis l'ordonnance "Colbert» de 1669. Le moment Turgot est aussi celui de tentatives innovantes, avec des commissions savantes omniprésentes, comme l'expérience autour de Condorcet qui bouleverse fondamentalement, voire s'oppose, aux procédures précédemment établies par les Ponts et Chaussées. La chute du ministère libéral emporte avec elle la plupart de ces démarches novatrices.

8 Le chapitre 5 insiste sur le foisonnement intellectuel né de la multiplication des écrits consacrés à la navigation au cours des trois décennies précédant la Révolution française. Cette fulgurante reprise des débats passe souvent par la promotion des canaux, plus que par l'aménagement des voies naturelles. Le but non dissimulé de ces écrits est d'inciter le gouvernement à agir. Avec d'autres acteurs, l'Académie royale des Sciences et les académies provinciales servent de relais au débat d'idées qui porte sur la gouvernance des infrastructures. 
9 Le chapitre suivant rappelle qu'à la fin de l'Ancien Régime, le réseau reste une idée et non une politique. La multiplication des projets se traduit toutefois par le perfectionnement des circuits décisionnels. Un passionnant travail de coordination des dossiers est opéré de concert par les administrateurs et les spécialistes. Les procédures d'expertise progressent, se normalisent, voire se ritualisent. Elles marquent notamment le retour en force des ingénieurs des Ponts et Chaussées pour l'évaluation technique des projets. Pour autant, les conflits sont nombreux: entre les bureaux ministériels, entre Paris et la province, entre les techniciens et les commerçants. En outre, l'instabilité ministérielle caractéristique de la fin du régime perturbe les travaux dédiés à la navigation, tout comme ceux des routes, même si l'incidence est moins marquée dans le second cas.

Les deux derniers chapitres abordent la naissance - tardive - d'une politique publique en faveur des navigations naturelles et artificielles. L'auteur décrit une à une les arcanes de cette politique, avec ses modalités de financement, les modes d'entretien, la police de la navigation, comme le bilan des travaux. Si les progrès sont indéniables, ils interviennent sur le tard, alors que l'édifice administratif se délite. En outre, ils restent bien en-deçà de l'effort fourni outre-Manche. Dans ce domaine, la France n'a donc pas la place de modèle qu'elle occupe pour le fait routier.

11 En forme d'épilogue, l'auteur insiste sur la rupture introduite par la Révolution française. La période des troubles balaye la majeure partie des procédures administratives mises en place en matière de navigation, une fois de plus à l'inverse de la route dont les démarches déjà en place font presque objet de consensus. La Révolution marque le retour du débat séculaire entre le régime de concession et la prise en charge directe par l'État. Elle marque aussi l'arrêt des chantiers ou des projets en cours, ainsi qu'une remise à plat du droit des voies navigables.

$12 \mathrm{Au}$ total, le travail d'Éric Szulman comble un important fossé historiographique qui faisait la part belle à la politique routière. Dans le domaine de la navigation, les réalisations, certes moins nombreuses, attirent moins l'attention des historiens. Pourtant, comme le démontre cette étude, cela se fait au détriment d'une histoire intellectuelle féconde qui met en jeu tous les cercles décisionnels de la seconde modernité. C'est une histoire beaucoup plus large qui est offerte, largement dédiée à l'organisation de l'État et à la bureaucratisation de ses services. Ainsi, cette plongée passionnante dans l'histoire de la navigation intérieure explore la façon dont l'État se structure et se transforme pour répondre aux multiples nécessités du moment. L'ensemble est complété par une impressionnante série de sources, une bibliographie actualisée, plus une série de précieux tableaux, schémas récapitulant les connexions administratifs ou cartes dont on regretterait presque qu'ils ne tiennent pas une place encore plus importante. 


\section{AUTEURS}

STÉPHANE BLOND

Université d'Evry-Val d'Essonne-IDHE.S 